\title{
CORAL-DMOEA: Correlation Alignment-Based Information Transfer for Dynamic Multi-Objective Optimization (Student Abstract)
}

\author{
Li Chen, Hua Xu \\ ${ }^{1}$ State Key Laboratory of Intelligent Technology and Systems, \\ Department of Computer Science and Technology, Tsinghua University, Beijing 100084, China \\ ${ }^{2}$ Beijing National Research Center for Information Science and Technology(BNRist), Beijing 100084, China \\ chenli19@mails.tsinghua.edu.cn, xuhua@tsinghua.edu.cn
}

\begin{abstract}
One essential characteristic of dynamic multi-objective optimization problems is that Pareto-Optimal Front/Set (POF/POS) varies over time. Tracking the time-dependent $\mathrm{POF} / \mathrm{POS}$ is a challenging problem. Since continuous environments are usually highly correlated, past information is critical for the next optimization process. In this paper, we integrate CORAL methodology into a dynamic multi-objective evolutionary algorithm, named CORAL-DMOEA. This approach employs CORAL to construct a transfer model which transfer past well-performed solutions to form an initial population for the next optimization process. Experimental results demonstrate that CORAL-DMOEA can effectively improve the quality of solutions and accelerate the evolution process.
\end{abstract}

\section{Introduction}

Dynamic Multi-objective Optimization Problems (DMOPs) are more challenging than static ones. A converged population is hard to adapt to a changing environment in time as it loses the exploration ability (Qian et al. 2017). Restarting optimization may be straightforward but ineffective. Usually, a new environment is strongly relevant to the previous one, so reusing experience has enormous potential to accelerate the next optimization process.

In recent years, transfer learning method has gradually aroused considerable interest. For instance, (Jiang et al. 2018) proposed Tr-DMOEA, which combines TCA with DMOEA. (Liu et al. 2019) proposed a Neural Networkbased Information Transfer method (NNIT) which learns how the environment changes. However, all these methods need to fine-tune the hyperparameters during the optimization process.

In this paper, we propose an algorithmic framework, which integrates a non-parametric domain adaptation method, CORrelation ALignment, into DMOEA, named CORAL-DMOEA.

\section{Proposed Algorithm}

CORAL-DMOEA is described in Algorithm 1. Once the environment changes, CORAL based Initial Population Gener-

Copyright (C) 2020, Association for the Advancement of Artificial Intelligence (www.aaai.org). All rights reserved.
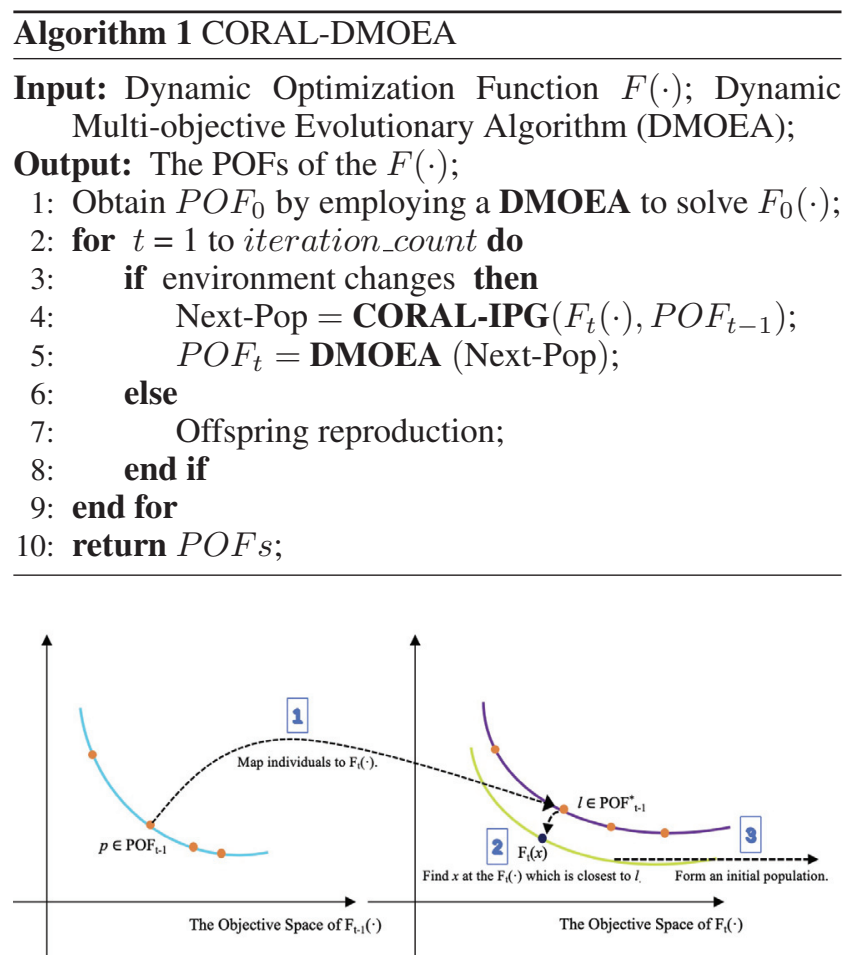

Figure 1: The key steps of CORAL-IPG.

ator (CORAL-IPG) establishes an initial population for the next evolutionary process. Figure 1 illustrates the key steps of CORAL-IPG. Step 1 maps individuals in the obtained $P O F_{t-1}$ to the objective space of $F_{t}(\cdot)$, named $P O F_{t-1}^{*}$. Afterwards, for each $l \in P O F_{t-1}^{*}$, Step 2 finds the corresponding $x \in F_{t}(\cdot)$ which is the closest to $l$. Finally, Step 3 outputs the set of corresponding individuals as the initial population. This figure represents the operation process from Line 3 to Line 8 of Algorithm 2.

CORAL uses covariance to evaluate the distances between different environments and aligns the data distribution in the past with the one in the current via minimizing the covariances. In the new objective space, the data feature of the past and new environments obey similar distributions as far 


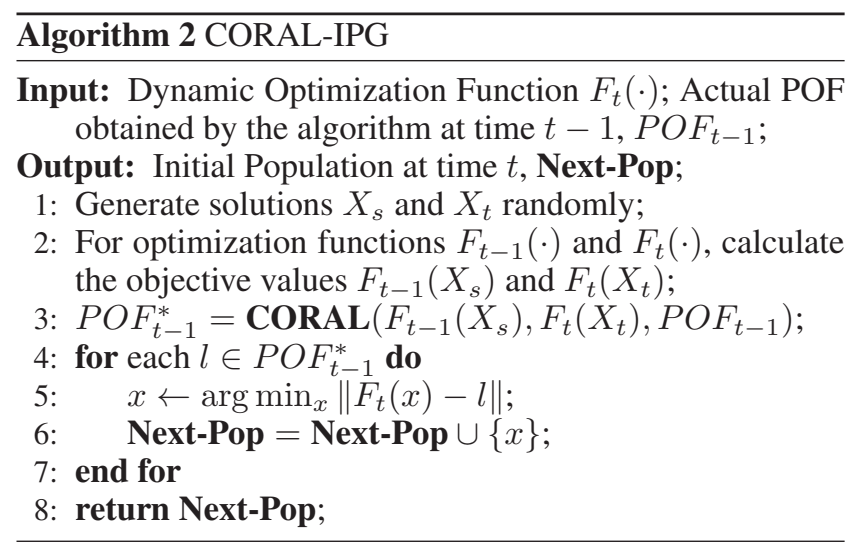

as possible.

Furthermore, the output of CORAL-IPG is an initial population. Thus we can combine any population-based DMOEA with CORAL-IPG to get a CORAL based dynamic evolutionary algorithm.

\section{Empirical Study}

We employ the IEEE CEC 2015 benchmark problem (Helbig and Engelbrecht 2015) to investigate the performances under different frequency changes. The frequency of change $\tau_{t}$ is set to $25,50,100$, and 200 respectively, and the corresponding iteration count $\tau$ is 500, 1000, 2000, and 4000 . Each function changes 20 times during a run, and each algorithm runs 30 times on each test function. Before the first change occurs, each algorithm runs 300 generations, and the population size is 300 .

MIGD and MHV are comprehensive performance metrics which provide an overall assessment of the convergence and diversity of algorithms. Table 1 illustrates the ratio of change (ROC) between DMOEAs and CORAL-DMOEAs on MIGD and MHV metrics. The best metric values are highlighted in the boldface.

We analyze the experimental results as a whole and draw the following conclusions. The overall effective rate of the CORAL-DMOEAs in MIGD metric is $87.50 \%$ (i.e., 21 cases perform well in 24 test functions). The results in FDA4, DMOP2_dec, and HE2 perform excellently. They belong to different DMOP types (Helbig and Engelbrecht 2015), which means that the proposed algorithm works on all types of DMOPs. The overall effective rate of the CORAL-DMOEAs in MHV metric is $91.67 \%$. However, the results in FDA5_iso and DMOP2_iso do not behave well, which means that the proposed algorithm may not be suitable for the isolated functions. Thus, CORAL can significantly improve the performances of the DMOEAs.

\section{Conclusion and Future Work}

In this paper, we propose an algorithmic framework CORAL-DMOEA. The combination of CORAL and DMOEAs accelerates the re-convergence of the population via reusing experience. Besides, the advantages of DMOEAs for solving DMOPs are preserved. The experimental results prove the superiority of CORAL-DMOEA.

\begin{tabular}{lcc}
\hline ROC $(\%)$ & CORAL-MOEA/D & CORAL-NSGA-II \\
\hline FDA4 & $\mathbf{8 0 . 1 0}$ & $\mathbf{7 9 . 5 6}$ \\
FDA5 & $\mathbf{2 0 . 5 3}$ & $\mathbf{1 5 . 0 1}$ \\
FDA5_iso & $\mathbf{1 6 . 0 1}$ & $\mathbf{9 7 . 0 2}$ \\
FDA5_dec & $\mathbf{5 0 . 9 2}$ & $\mathbf{5 0 . 2 8}$ \\
DIMP2 & $\mathbf{6 6 . 8 5}$ & $\mathbf{2 4 . 4 5}$ \\
DMOP2 & $\mathbf{1 . 1 3}$ & $\mathbf{1 3 . 0 9}$ \\
DMOP2_iso & $\mathbf{1 . 5 9}$ & -9.06 \\
DMOP2_dec & $\mathbf{8 9 . 8 2}$ & $\mathbf{8 1 . 8 6}$ \\
DMOP3 & -0.79 & $\mathbf{7 3 . 2 9}$ \\
HE2 & $\mathbf{9 5 . 0 3}$ & $\mathbf{8 6 . 0 6}$ \\
HE7 & $\mathbf{5 2 . 8 5}$ & $\mathbf{6 0 . 3 8}$ \\
HE9 & $\mathbf{1 3 . 3 3}$ & -6.86 \\
\hline
\end{tabular}

(a) ROC values of CORAL-DMOEAs on MIGD

\begin{tabular}{lrr}
\hline ROC $(\%)$ & CORAL-MOEA/D & CORAL-NSGA-II \\
\hline FDA4 & $\mathbf{1 0 4 . 5 6}$ & $\mathbf{2 3 2 7 . 1 6}$ \\
FDA5 & $\mathbf{2 . 8 6}$ & $\mathbf{7 0 . 2 4}$ \\
FDA5_iso & $\mathbf{3 . 0 4}$ & -14.39 \\
FDA5_dec & $\mathbf{3 2 5 . 3 2}$ & $\mathbf{4 6 9 3 . 9 2}$ \\
DIMP2 & $\mathbf{2 4 3 5 . 1 2}$ & $\mathbf{2 0 1 9 . 0 4}$ \\
DMOP2 & $\mathbf{2 1 2 . 2 5}$ & $\mathbf{1 2 2 . 0 4}$ \\
DMOP2_iso & $\mathbf{0 . 4 3}$ & $\mathbf{0 . 8 3}$ \\
DMOP2_dec & $\mathbf{1 0 6 5 . 3 8}$ & $\mathbf{2 1 5 . 4 0}$ \\
DMOP3 & $\mathbf{9 6 . 9 8}$ & $\mathbf{7 1 . 5 3}$ \\
HE2 & $\mathbf{5 5 4 . 5 0}$ & $\mathbf{1 5 6 . 0 7}$ \\
HE7 & $\mathbf{3 0 . 5 3}$ & $\mathbf{2 6 . 9 3}$ \\
HE9 & $\mathbf{2 5 . 8 2}$ & -5.80 \\
\hline
\end{tabular}

(b) ROC values of CORAL-DMOEAs on MHV

Table 1: The comparison between DMOEAs and CORALDMOEAs (ROC means the ratio of change)

In future, we will employ the CORAL-DMOEA in practical application.

\section{Acknowledgment}

This paper is funded by the National Natural Science Foundation of China (Grant No: 61673235) and National Key R\&D Program Projects of China (Grant No: 2018YFC1707605).

\section{References}

Helbig, M., and Engelbrecht, A. 2015. Benchmark functions for cec 2015 special session and competition on dynamic multi-objective optimization. Technical Report.

Jiang, M.; Huang, Z.; Qiu, L.; Huang, W.; and Yen, G. G. 2018. Transfer learning-based dynamic multiobjective optimization algorithms. IEEE Transactions on Evolutionary Computation 22(4):501-514.

Liu, X.-F.; Zhan, Z.-H.; Gu, T.-L.; Kwong, S.; Lu, Z.; Duh, H. B.-L.; and Zhang, J. 2019. Neural network-based information transfer for dynamic optimization. IEEE Transactions on Neural Networks and Learning Systems.

Qian, S.; Ye, Y.; Jiang, B.; and Xu, G. 2017. A microcloning dynamic multiobjective algorithm with an adaptive change reaction strategy. Soft Computing 21(13):37813801 . 